\title{
ORIGINAL ARTICLE \\ Functional MRI of the cortical sensorimotor system in patients with hereditary spastic paraplegia
}

\author{
T Tomberg ${ }^{1,2,6}, \mathrm{M}$ Braschinsky ${ }^{3,6}, \mathrm{~K}$ Rannikmäe ${ }^{3}, \mathrm{~J} \mathrm{Kepler}^{4}, \mathrm{~K} K e p l e r^{5}, \mathrm{~J} \mathrm{Kõrv}^{3}$, Ü Linnamägi ${ }^{3}$ and T Asser $^{3}$
}

Objectives: The study aimed to use functional magnetic resonance imaging ( $\mathrm{FMRI}$ ) to ascertain changes in sensorimotor system function in patients with hereditary spastic paraplegia (HSP) and to correlate it with severity of spasticity and paresis.

Setting: Tartu University Hospital, Tartu, Estonia.

Methods: Nine patients with autosomal-dominant pure HSP and 14 age- and sex-matched healthy controls were investigated with a 1.5T fMRI scanner during flexion/extension of the right-hand fingers and right ankle. Images were analysed with a general linear model and Statistical Parametrical Mapping software. Highest Z-scores were identified from probability maps, and weighted laterality indices were calculated using combined bootstrap/histogram analysis; these were correlated with clinical severity of spasticity and paresis. Results: During hand movements, clusters located in contralateral primary sensorimotor and premotor areas activated in both controls and patients. Bilateral activation occurred in the supplementary motor area, parietal operculum (predominantly contralateral) and cerebellum (predominantly ipsilateral). During the ankle task, bilateral activation was noted in the primary sensorimotor area, supplementary motor area and cerebellum. Activation clusters in HSP patients were smaller than those in controls in the sensorimotor area, especially during the ankle task, and more pronounced ipsilaterally in cerebellum both during hand and ankle motor tasks. Spasticity was significantly associated with contralateral activation in the sensory area and correlated negatively with the highest Z-scores in Brodmann areas 1-2-3 and 4.

Conclusion: Our results suggest changes in cortical sensorimotor network function in patients with HSP compared with healthy subjects. Lower activation in patients might reflect damage to the corticospinal tract, be influenced by compensatory mechanisms, and/or be a reflection of neurorehabilitation.

Spinal Cord (2012) 50, 885-890; doi:10.1038/sc.2012.70; published online 3 July 2012

Keywords: cortical reorganisation; functional magnetic resonance imaging; hereditary spastic paraplegia; Strümpell-Lorrain disease

\section{INTRODUCTION}

Hereditary spastic paraplegia (HSP) or the Strümpell-Lorrain disease is a group of rare chronic neurodegenerative disorders marked by clinical and genetic heterogeneity. It has been classified into two forms, 'pure' (pHSP) and 'complex' (cHSP). pHSP presents with spasticity and motor deficit in the legs, brisk reflexes and Babinski's signs; deep sensory impairment and sphincter disturbances are also common. For cases of cHSP, other neurological or extra-neurological features can be present. ${ }^{1}$ The disease affects substantially health related quality of life of persons with HSP. ${ }^{2}$

The primary pathology in HSP is axonal degeneration that is maximal at the distal ends of the corticospinal tracts and at the distal ends of dorsal column fibres. ${ }^{3}$ Previous studies in HSP patients have revealed cerebral involvement as well, including cognitive decline, white matter abnormalities, involvement of motor cortex and alterations of regional cerebral blood flow and brain activity. ${ }^{4-8}$ However, the pattern of possible cortical functional reorganisation and its correlates with motor impairments are yet poorly understood.

The objectives of this study were to ascertain changes in sensorimotor system function in patients with HSP by using functional magnetic resonance imaging ( $\mathrm{fMRI}$ ) and to correlate fMRI changes with the severity of spasticity and paresis in legs.

\section{MATERIALS AND METHODS}

\section{Subjects}

Patient data were acquired from an epidemiological study database consisting of 59 persons with HSP. ${ }^{9}$ Patients with autosomal-dominant pHSP (ADpHSP) and mutations in the SPAST gene (also known as the SPG4 gene-the most frequent genetic pathology found in HSP worldwide in general and in Estonia in particular) were invited to participate in the study as were their affected family members with the same phenotype. Other Estonian patients with HSP were not included in this study in order to ensure the genetic and clinical homogeneity of the study group. Nine right-handed patients (5 women, 4 men, mean age 47 years, age range 22-69 years) and 14 age- and sex-matched right-handed healthy controls ( 8 women, 6 men, mean age 47 years, age range $22-70$ years) consented for participation.

All nine patients studied had a positive family history for HSP and belonged to three different pedigrees (Table 1). Five patients had pathogenic mutations in the SPAST gene, and two patients had non-pathogenic mutations in the SPAST gene. One patient had no changes in the SPAST gene but belonged to a pedigree with other affected members with non-pathogenic mutation. One patient refused to participate in genetic testing but belonged to a larger family

${ }^{1}$ Department of Neurology and Neurosurgery, University of Tartu, Tartu, Estonia; ${ }^{2}$ Department of Radiology, Tartu University Hospital, Tartu, Estonia; ${ }^{3}$ Department of Neurology and Neurosurgery, University of Tartu, Tartu, Estonia; ${ }^{4}$ Diagnostics Service, Pärnu Hospital, Pärnu, Estonia and ${ }^{5}$ nnstitute of Physics, University of Tartu, Tartu, Estonia

${ }^{6}$ These authors contributed equally to this work.

Correspondence: Dr M Braschinsky, Department of Neurology, University of Tartu, Puusepa 8H, Tartu 51014, Estonia.

E-mail: mark.braschinsky@kliinikum.ee

Received 20 February 2012; revised 20 April 2012; accepted 11 May 2012; published online 3 July 2012 
with pathogenic mutation in the SPAST gene, where all affected members had the same phenotype of the disease. ${ }^{10}$ Eight included patients had lower spastic paraparesis with different degree of spasticity and paresis (Table 1). One man was asymptomatic but had pyramidal signs present—his data were analysed also separately. Four patients used antispasticity medications (tizanidine or baclofen) before the study, but were taken off the medication for 3 days before the time of the study.

Spasticity was evaluated using the Modified Ashworth Scale (MAS) to assess the antagonist muscles: hamstrings, thigh adductor, gastrocnemius and soleus. ${ }^{11}$ A $0-5$ grading system was applied (0, no increase in muscle tone; 5 , affected part rigid). ${ }^{11}$ Severity of paresis of the lower limbs was assessed using on the Medical Research Council (MRC) Scale for Muscle Strength grading it from $0-5(5$, normal strength; 0 , no movement $) .{ }^{12}$

\section{Functional MRI scanning paradigm}

Before examination, the subjects were informed about the procedure, tasks and length of the examination. A block design with six alternating task-rest cycles was used, starting with a rest cycle. Two paradigms were used for sensorimotor function assessment: (1) flexion/extension of the right-hand fingers; and (2) flexion/extension of the right ankle. Brief verbal instructions to move or rest were given. Before the experiment, all subjects were trained to perform movements at a frequency of $1 \mathrm{~Hz}$. Performance of the task was monitored visually, and the number of movements was registered. The frequency of movements ( \pm s.d.) in patients (hand task $0.93 \pm 0.26 \mathrm{~Hz}$; ankle task $0.92 \pm 0.33 \mathrm{~Hz}$ ) and controls (hand task $0.86 \pm 0.18 \mathrm{~Hz}$; ankle task $0.86 \pm 0.20 \mathrm{~Hz}$ ) did not differ significantly (Wilcoxon two-sample test).

Table 1 Basic characteristics of the nine patients with positive family history for hereditary spastic paraplegia

\begin{tabular}{|c|c|c|c|c|c|c|}
\hline Pedigree & Sex & $\begin{array}{l}\text { Agelage } \\
\text { at onset } \\
\text { (years) }\end{array}$ & $\begin{array}{l}\text { Brain MRI } \\
\text { findings }\end{array}$ & $\begin{array}{l}\text { MAS } \\
\text { score }\end{array}$ & $\begin{array}{l}\text { MRC Scale for } \\
\text { muscle strength } \\
\text { paresis severity } \\
\text { score }\end{array}$ & $\begin{array}{l}\text { Mutations } \\
\text { in the } \\
\text { SPAST } \\
\text { gene }\end{array}$ \\
\hline I & $\mathrm{F}$ & $54 / 35$ & Normal & 3 & 4 & $\mathrm{P}$ \\
\hline I & $\mathrm{F}$ & $22 / 10$ & Normal & 2 & 5 & $\mathrm{P}$ \\
\hline I & $M$ & $36 / 16$ & Normal & 4 & 5 & NK \\
\hline II & $\mathrm{F}$ & $63 / 40$ & Normal & 4 & 1 & $\mathrm{P}$ \\
\hline II & $\mathrm{F}$ & $44 / 12$ & Normal & 5 & 1 & $\mathrm{P}$ \\
\hline II & $M$ & $38 / 21$ & Normal & 3 & 5 & $\mathrm{P}$ \\
\hline III & $\mathrm{F}$ & $69 / 57$ & $\begin{array}{l}\text { Moderate brain } \\
\text { atrophy, thinning } \\
\text { of } \mathrm{CC} \text {, leucopathy }\end{array}$ & 2 & 5 & NP \\
\hline III & $M$ & $32 / N A$ & Normal & 0 & 5 & NP \\
\hline III & M & $65 / 32$ & Mild leucopathy & 1 & 4 & NC \\
\hline
\end{tabular}

Abbreviations: CC, corpus callosum; F, female; M, male; MAS, Modified Ashworth Scale (measured muscles are hamstrings, thigh adductor, gastrocnemius, and soleus); MRC, Medical Research Council; NC, no change; NK, not known; NP, non-pathogenic; P, pathogenic.

\section{Image acquisition}

Images were obtained on a 1.5-T MR scanner (Magnetom Symphony; Siemens Medical Systems, Erlangen, Germany). Before obtaining functional scans, a high-resolution T1-weighted anatomical image was obtained with the gradient echo, fast low-angle shot sequence (repetition time/echo time $(\mathrm{TR} / \mathrm{TE})=12 /$ $5.68 \mathrm{~ms}$, flip angle $15^{\circ}$, resolution $224 \times 256$, voxel size $1 \times 1 \times 1 \mathrm{~mm}^{3}, 176$ sagittal planes). Functional $\mathrm{T} 2{ }^{\star}$-weighted images were obtained using the gradient echo, echo-planar imaging sequence $(\mathrm{TR} / \mathrm{TE}=4 / 50 \mathrm{~ms}$, resolution $64 \times 64$, voxel size $3 \times 3 \times 3 \mathrm{~mm}^{3}$, slice gap $0.75 \mathrm{~mm}, 36$ axial planes, interleaved scan). Altogether, 60 whole-brain functional images were obtained for each patient and control subject while the subjects were performing the described tasks.

\section{Image processing}

Image processing was performed using Statistical Parametrical Mapping software (SPM8; Wellcome Trust Centre for Neuroimaging, London, UK), which is a suite of MATLAB (The MathWorks, Inc., Natick, MA, USA) functions to process and analyse functional neuroimaging data. ${ }^{13}$

First step of spatial pre-processing was realignment of functional images, where movement effects were discounted. Then high-resolution anatomical images were co-registered with functional images to maximise the mutual information. Pre-processing continued with segmentation of high-resolution anatomical images, where the Montreal Neurological Institute 452 white matter, grey matter and cerebrospinal fluid probability maps were used to yield a parametric description for normalisation. ${ }^{14}$ In normalisation processing, images were also bias corrected. Image pre-processing ended with smoothing by $8 \times 8 \times 8 \mathrm{~mm}^{3}$ full-width at half maximum isotropic Gaussian kernel.

\section{Statistical analysis}

Image processing was followed by a general linear model-based statistical analysis of the functional images. Multiple comparisons problem was corrected by masking images with Brodmann area (BA) masks (Figure 1) from MRIcro and by doing region-of-interest analysis using WFU PickAtlas. ${ }^{15-17}$ Unmasked t-maps were divided into two groups (HSP patients and healthy controls) and were taken onto second level, that is, random effect analysis. One-sample $t$-test was used to explore group-specific activations. The resulting t-maps for each subject were analysed within BA 1-2-3, BA 4 and BA 6, defined by MRIcro templates (www.mricro.com) (Figure 1). The highest $Z$-scores were identified from probability maps, and weighted laterality indices (wLI) were calculated using combined bootstrap/histogram analysis after Wilke and Schmithorst; these were correlated with clinical severity of spasticity and paresis. ${ }^{18}$ The $\mathrm{wLI}<-1$ corresponds to right-hemispheric, wLI- $0.2 \ldots 0.2$ to bilateral and wLI $>0.2$ to left-hemispheric brain activation. Images were thresholded at a given value $(t)$, and only voxels at which all images exceed the threshold were included.

Inter- and intrasubject reproducibility of the used methodology has been assessed previously. ${ }^{19}$

Additional statistical analysis was performed using the statistical package SAS Version 9.1. Differences between the groups were studied with the

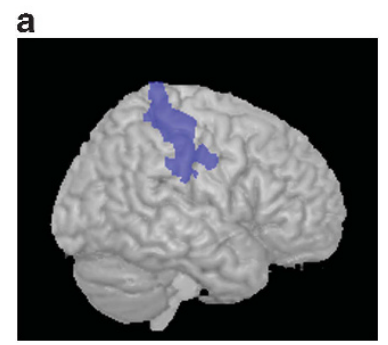

b

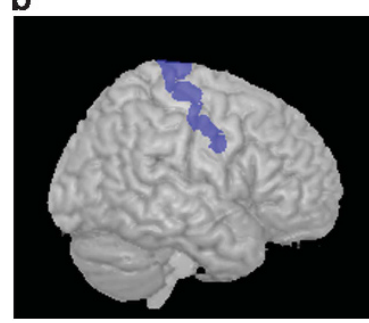

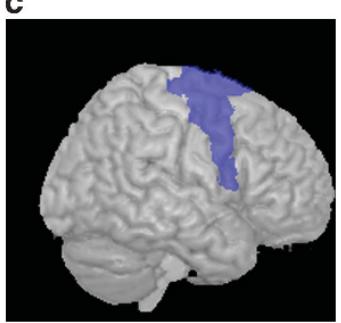

Figure 1 BA masks (regions of interest) are defined on a normalised Montreal Neurological Institute single_subject_T1 template using modified BA templates by MRIcro: (a) BA 1-2-3; (b) BA 4; (c) BA 6 . 
nonparametric Mann-Whitney $U$-test. The Kolmogorov-Smirnov criterion was employed for assessment of normality. To examine the association between the variables, the Spearman's correlation test was used. All $P$ values were twosided, and differences were considered statistically significant if $P$ values were less than 0.05 .

\section{Statement of ethics}

We certify that all applicable institutional and governmental regulations concerning the ethical use of human volunteers were followed during the course of this research. This study was approved by the Ethics Review Committee on Human Research of the University of Tartu, and informed consent was obtained from all study participants.

\section{RESULTS}

During hand movements, clusters located in the contralateral primary sensorimotor and premotor areas activated in both the control subjects and the patients (Table 2, Figure 2).

Bilateral activation occurred in the supplementary motor area, in the parietal operculum (predominantly contralateral) and in the cerebellum (predominantly ipsilateral).

During the ankle task, bilateral activation was noted in the primary sensorimotor area, in supplementary motor area and in the cerebellum. Activation clusters in HSP patients were smaller than those in controls in the sensorimotor area, especially during the ankle task, and more pronounced ipsilaterally in the cerebellum during both the hand and ankle motor tasks.

During the ankle task, less lateralised activation in BA 4 occurred in patients than in controls $(P<0.1)$ (Table 3 ). In BA $1-2-3$ and BA 6 , activation was bilateral ( $\mathrm{wLI}=0.07-0.22$ ) both in patients and in controls. In control subjects, lateralisation was significantly more pronounced in BA 4 than in BA 1-2-3 and BA 6 during both the hand and ankle tasks $(P<0.001)$.

In patients with HSP, mean maximal $Z$-scores during the hand task, and especially during the ankle task, were lower than those in controls, but a statistically significant difference was revealed only in BA 1-2-3 $(P<0.02)$ during the ankle task (Table 3$)$.

Maximal $Z$-scores in BA $1-2-3$ and in BA 4 were negatively correlated with the scores for spasticity in leg muscles $(P<0.05)$ (Figure 3).
Spasticity significantly correlated with wLI only in BA 1-2-3 $(P<0.05)$, indicating that more severe spasticity was associated with contralateral activation in the sensory area (Figure 4).

Paresis did not correlate significantly with wLI and Z-scores. Also, no correlation was noted with either disease duration or patients' age or sex.

\section{DISCUSSION}

The results of our study suggest changes in cortical sensorimotor network function in patients with HSP compared with healthy subjects. Activation in the sensorimotor cortex was associated with spasticity, but not with the degree of paresis. The exact mechanism behind these findings is unknown, but it can be related to the clinical peculiarity of HSP that separates it from other causes of spastic paraparesis: it has been shown, that the spasticity contributes to gait disturbance disproportionally more than the paresis, with a notable discrepancy between the degrees of spasticity and of muscle weakness. ${ }^{20}$

It is difficult to compare our results with similar ones because, to our knowledge, there is only one published study on this topic that also used the same technique. ${ }^{7}$ It is also hard to find a disease to compare with that could be similar to HSP in terms of both underlying neuropathological process and clinical presentation (almost isolated pyramidal involvement with clear domination of spasticity over paresis and slow rate of progression). This is because, to date, the extent of cortical involvement in the pathogenesis of HSP and the pattern of possible cortical functional reorganisation with its correlates with motor impairment are poorly investigated. Nevertheless, despite the main pathological process of distal axonal degeneration, cortical involvement was studied previously in a few works that used different techniques. ${ }^{4-8}$

Erichsen et al. ${ }^{4}$ used magnetic resonance spectroscopy on a small but genetically well-defined group of SPAST-related HSP patients. The cholin/creatin ratio in motor cortex of the patients was significantly lower than that in controls and was significantly associated with agerelated verbal learning and memory reduction. Authors concluded that these findings support involvement of motor cortex in HSP.

Duning et al. ${ }^{8}$ examined six patients with pHSP because of SPAST mutations by using conventional MRI, diffusion tensor imaging and

Table 2 Statistically significant clusters of activation in Brodmann areas 1-2-3, 4 and 6 for controls and patients $(n=8)$

\begin{tabular}{|c|c|c|c|c|c|}
\hline Group/task & t-value (threshold) & Cluster volume $\left(\mathrm{cm}^{3}\right)$ & MNI coordinates of the maximum voxel $x, y, z$ & Location & P-value (uncorrected) \\
\hline \multirow[t]{3}{*}{ Controls' hand task } & 3.85 & 14.6 & $-39,-22,55$ & BA $1-2-3$ & $<0.001$ \\
\hline & 3.85 & 6.5 & $-39,-22,55$ & BA 4 & $<0.001$ \\
\hline & 3.85 & 17.3 & $-36,-22,63$ & BA 6 & $<0.001$ \\
\hline \multirow[t]{3}{*}{ Controls' ankle task } & 3.85 & 8.8 & $-57,-37,33$ & BA $1-2-3$ & $<0.001$ \\
\hline & 3.85 & 10.1 & $0,-19,59$ & BA 4 & $<0.001$ \\
\hline & 3.85 & 48.6 & $3,-7,63$ & BA 6 & $<0.001$ \\
\hline \multirow[t]{3}{*}{ Patients' hand task } & 4.79 & 4.9 & $-36,-19,51$ & BA $1-2-3$ & $<0.001$ \\
\hline & 4.79 & 3.6 & $-39,-16,55$ & BA 4 & $<0.001$ \\
\hline & 4.79 & 4.7 & $-39,-13,51$ & BA 6 & $<0.001$ \\
\hline \multirow[t]{3}{*}{ Patients' ankle task } & 1.89 & 0.3 & $-39,-40,59$ & BA $1-2-3$ & $<0.05$ \\
\hline & 1.89 & 3.7 & $0,-25,63$ & BA 4 & $<0.05$ \\
\hline & 1.89 & 3.4 & $0,-16,70$ & BA 6 & $<0.05$ \\
\hline
\end{tabular}

Abbreviations: BA, Brodmann area; MNI, Montreal Neurological Institute. 

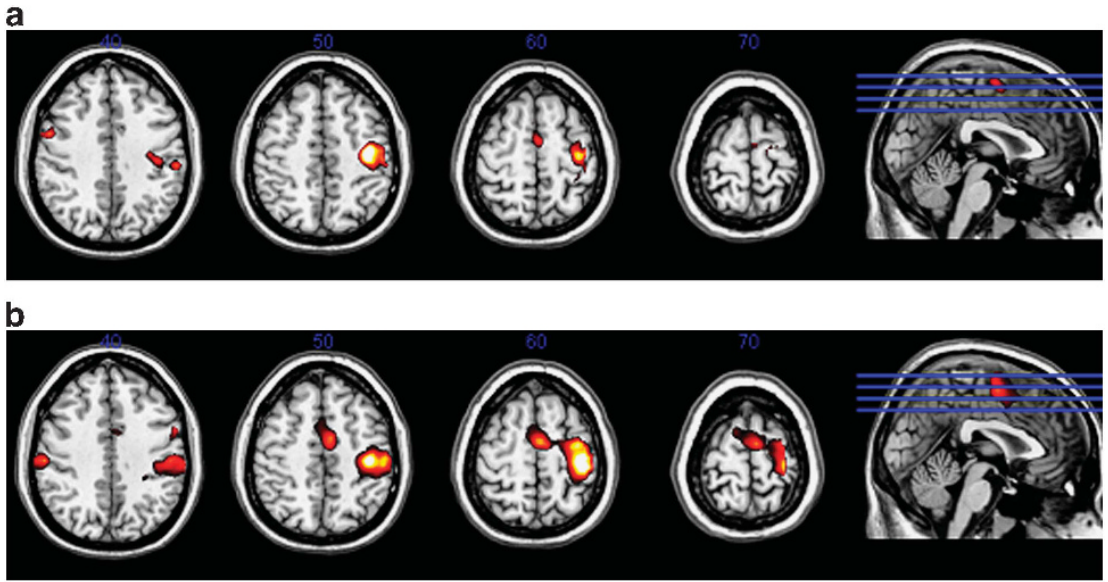

c

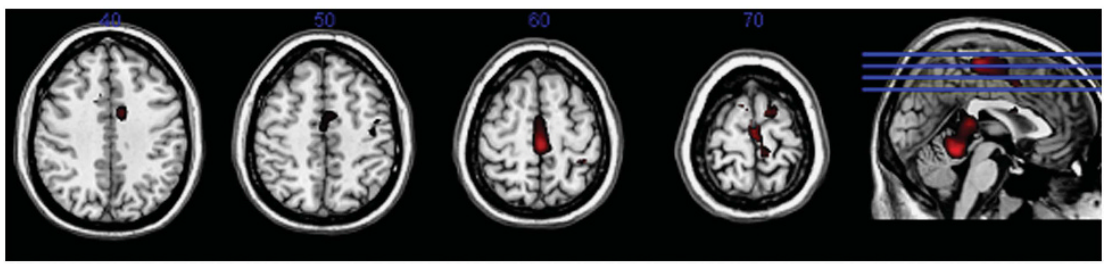

d

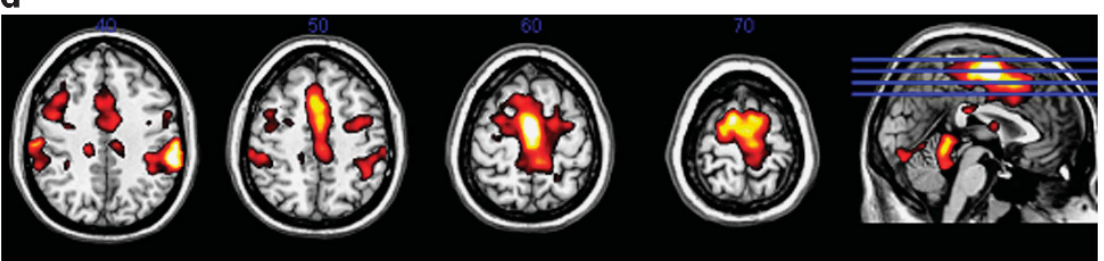

Figure 2 Group activation maps (random effect analysis). (a) Patients' hand task $(P<0.001)$, (b) controls' hand task $(P<0.001)$, (c) patients' ankle task $(P<0.05)$ and $(\mathbf{d})$ controls' ankle task $(P<0.001)$. Right side of the figure corresponds to the left side of the brain.

Table 3 Means of weigted laterality indices ( $w L I)$ and maximum $Z$-scores corresponding to Brodmann areas (BA) in HSP patients and controls during hand and ankle tasks

\begin{tabular}{lll}
\hline BAltask & $w L I$ (mean \pm s.d.) & Z-score (mean \pm s.d.) \\
\hline BA 1-2-3 & & \\
Controls' hand task & $0.75 \pm 0.03$ & $6.27 \pm 0.45$ \\
Controls' ankle task & $0.07 \pm 0.11$ & $5.21 \pm 0.33^{* *}$ \\
Patients' hand task & $0.71 \pm 0.02$ & $3.59 \pm 0.59$ \\
Patients' ankle task & $0.28 \pm 0.23$ & \\
& & \\
BA 4 & & $6.59 \pm 0.42$ \\
Controls' hand task & $0.89 \pm 0.02$ & $5.64 \pm 0.49$ \\
Controls' ankle task & $0.60 \pm 0.03^{*}$ & $5.86 \pm 0.57$ \\
Patients' hand task & $0.84 \pm 0.04$ & $4.41 \pm 0.37$ \\
Patients' ankle task & $0.35 \pm 0.17$ & \\
BA 6 & & $6.62 \pm 0.45$ \\
Controls' hand task & $0.69 \pm 0.04$ & $5.40 \pm 0.34$ \\
Controls' ankle task & $0.16 \pm 0.07$ & $6.07 \pm 0.52$ \\
Patients' hand task & $0.69 \pm 0.04$ & $4.39 \pm 0.59$ \\
Patients' ankle task & $0.19 \pm 0.15$ & \\
\end{tabular}

Abbreviation: HSP, hereditary spastic paraplegia.

${ }^{*} P<0.1 . * * P<0.05$, statistical significant difference compared with patients (Wilcoxon twosample test).
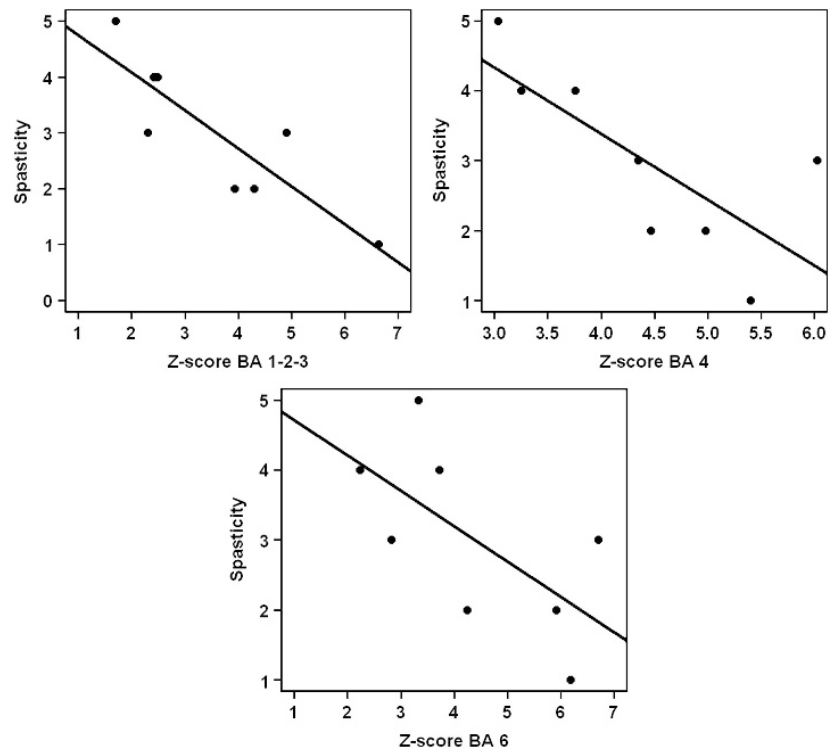

Figure 3 Scatterplot of maximal Z-scores according to the $\mathrm{BA}$ and scores for spasticity with regression line: (a) BA 1-2-3 $(r=-0.788$, $P=0.020)$; (b) BA $4(r=-0.800, P=0.017)$; and (c) BA $6(r=-0.630$, $P=0.093)$. 


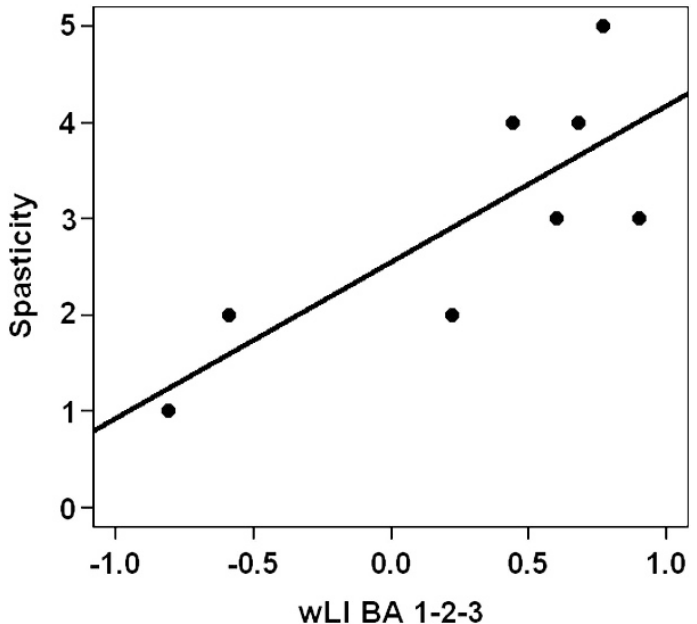

Figure 4 Scatterplot of $w \mathrm{LI}$ in BA 1-2-3 and scores for spasticity with regression line $(r=0.751, P=0.031)$.

brain volumetry. The only pathology found was revealed by diffusion tensor imaging: widespread disturbance of white matter integrity, mainly affecting the corticospinal tract. This finding is consistent with the clinical representation of the disorder, but probably not specific to HSP.

A Danish group published two articles on the same cohort of HSP patients in order to investigate the extent of motor cortical functional reorganisation in patients with SPAST-related HSP, by using positron emission tomography. ${ }^{5,6}$ They found that the patients with HSP had significantly decreased regional cerebral blood flow in the left frontotemporal cortex at resting state and that patients had a tendency toward more widespread activation in sensorimotor cortical and cerebellar regions upon movements (right ankle flexion-extension and right shoulder flexion-extension were studied). Although statistically significant differences were found only for the ankle movement response, patients showed significantly increased regional cerebral blood flow in the right and left primary motor cortices, the supplementary motor areas and the right premotor cortex compared with controls.

The hypothesis of an impaired function was investigated using neurophysiological methods as well. For instance Satrucci et al..$^{21}$ used short latency somatosensory evoked potentials in patients with SPAST-related AD-pHSP. Somewhat similarly to the present study authors showed that cortical somatosensory evoked potentials from lower limbs were abnormal and correlated with spasticity. But there were no abnormalities found in neurophysiological parameters from upper limbs.

To our knowledge, only one study has been published, in which, like the present study, fMRI was used to explore cortical activation: Koritnik et al. ${ }^{7}$ found that there was increased activation in bilateral posterior parietal cortex and left primary sensorimotor cortex in patients with HSP. As the authors used two different levels of difficulty for performing the selected motor movements, they rightfully concluded that these changes probably reflect reorganisation of the cortical motor system and not just increased activation because of a greater relative motor effort. Unfortunately, there were some methodological concerns in the study. First, the authors used a heterogeneous group of HSP patients, which affects the interpretation of the results. It is unclear at the present time to what extent having additional neurological features, as in cHSP, can influence the cortical activation. Second, in their study, only finger movements were selected as a motor task to evaluate cortical activation; however, patients with HSP have more pronounced motor deficit in legs than in arms, including both spasticity and paresis. ${ }^{22}$ Hence, it is important to include motor tests evaluating legs as well. Perhaps because of the latter, the authors also did not mention any correlation between spasticity and cortical activation, although spasticity was measured.

Our group included only patients with AD-pHSP; hence, it represents a homogeneous group of HSP patients. In fact our study group is genetically and clinically the most homogeneous of all similar studies published so far on this topic. We also temporary discontinued all antispastic medications to exclude the possibility of drugs affecting the results of the study, although the precise possible mechanism of influence of these medications on cortical activation is unknown. The motor tasks covered both upper and lower extremities. As, to date, it is unclear which of the main two neurological features influences cortical reorganisation morespasticity or paresis - these clinical correlates were investigated. As in HSP, spasticity dominates over paresis, it can be speculated that this might be a reason for the more significant correlations with cortical activation found in our study.

Lower activation in patients compared with controls might reflect damage to the corticospinal tract producing reduced motor output during activation, which is associated with lower afferent feedback and activity in sensory areas. It may also be influenced by the compensatory mechanism, cortical plasticity. ${ }^{23}$ It is not excluded that certain patterns of cortical activation can be a reflection of active neurorehabilitation. All these hypotheses are still to be answered in future studies specifically designed to do so and taking into consideration the fact that all possible mechanisms remain to be fully elucidated.

Interestingly, in the group of patients there was one person with neither complaints, nor paresis or spasticity, but with notable hyperreflexia in legs and bilateral Babinski's sign. He belongs to a larger pedigree of patients with the same mutation in the SPAST gene. ${ }^{10}$ This 32 year-old man probably didn't reach the age of the first notable deficit to appear clinically. When this person's results were analysed separately, a few interesting trends appeared. He had higher $Z$-scores for almost all the investigated movements, except for BA 1-2-3 $Z$-score $(4,14)$, which was somewhat smaller than in controls $(4,44)$, but higher than in patients $(3,65)$. On finger tasks this person's cortical activation was more lateralized. His results for the hand's motor tasks were resembling controls' ones. On the contrary, cortical activation during the motor tasks in legs is more suggestive for the pattern seen in the patients group. These findings might represent individual variations and are not conclusive. But such a trend may suggest that pathological process of cortical reorganisation probably starts before the symptomatic phase of the disease and initially is represented in legs' motor areas, correlating with clinical pattern of the initial presentation of HSP. This hypothesis is indirectly supported by the differences found during the hand task between controls and patients, who actually had no obvious motor deficit in upper extremities (in present study and one by Koritnik et al. ${ }^{7}$ ). This is one of the most interesting findings of our study and these trends have never been described in literature so far. Hence, further research can also concentrate on asymptomatic patients with known genetic pathology of HSP. If the results of such research could confirm our findings, this might represent the possibility of using fMRI for the preclinical stage of the disease and can possibly be used as a prognostic marker for the symptomatic phase of HSP. 


\section{Limitations}

There are some limitations associated with this study. The number of participants in this study is somewhat low. A larger study would have been more robust for statistical analyses, and therefore, more conclusive. Despite the clear-cut phenotype of AD-pHSP, not all patients had the pathogenic mutations in the SPAST gene. Hence, the results are not to be implemented strictly to the SPAST-related HSP. Somatosensory evoked potentials were not used because of the unavailability of the method in Estonia.

\section{CONCLUSION}

Our results suggest changes in cortical sensorimotor network function in patients with HSP compared with the healthy subjects. More studies to increase the conclusiveness of the data and to gain more evidence in the field of this subject are needed. It could represent a necessary preclinical basis for planning evidence-based neurorehabilitation of persons with HSP.

\section{DATA ARCHIVING}

There were no data to deposit.

\section{CONFLICT OF INTEREST}

The authors declare no conflict of interest.

\section{ACKNOWLEDGEMENTS}

We would like to thank Pille Kool for her assistance in statistical analysis.

This study was supported by Estonian Science Foundation research Grant no. 7868 and targeted financing from the Estonian Ministry of Education and Research (Grant no. SF0180064s07).

1 McDermott CJ, White K, Bushby K, Shaw P. Hereditary spastic paraparesis: a review of new developments. J Neurol Neurosurg Psychiatry 2000; 69: 150-160.

2 Braschinsky M, Rannikmäe K, Krikmann Ü, Lüüs S-M, Raidvee A, Gross-Paju K et al. Health-related quality of life in patients with hereditary spastic paraplegia in Estonia. Spinal Cord 2011; 49: 175-181.

3 Harding AE. Hereditary spastic paraplegias. Semin Neurol 1993; 13: 333-336.
4 Erichsen AK, Server A, Landrø NI, Sandvik L, Tallaksen CM. Proton magnetic resonance spectroscopy and cognition in patients with spastin mutations. Brain 2009; 132: 1577-1588

5 Scheuer KH, Nielsen JE, Krabbe K, Simonsen C, Koefoed P, Sørensen SA et al Reduced regional cerebral blood flow in SPG4-linked hereditary spastic paraplegia. J Neurol Sci 2005; 235: 23-32.

6 Scheuer KH, Nielsen JE, Krabbe K, Paulson OB, Law I. Motor activation in SPG4 linked hereditary spastic paraplegia. J Neurol Sci 2006; 244: 31-39.

7 Koritnik B, Azam S, Knific J, Zidar J. Functional changes of the cortical motor system in hereditary spastic paraparesis. Acta Neurol Scand 2009; 120: 182-190.

8 Duning T, Warnecke T, Schirmacher A, Schiffbauer H, Lohmann H, Mohammadi S et al. Specific pattern of early white-matter changes in pure hereditary spastic paraplegia. Mov Disord 2010; 25: 1986-1992.

9 Braschinsky M, Lüüs S-M, Gross-Paju K, Haldre S. The prevalence of hereditary spastic paraplegia and the occurrence of SPG4 mutations in Estonia. Neuroepidemiology 2009; 32: 89-93.

10 Braschinsky M, Tamm R, Beetz C, Sachez-Ferrero E, Raukas E, Lüüs SM et al. Unique spectrum of SPAST variants in Estonian HSP patients: presence of benign missense changes but lack of exonic rearrangements. BMC Neurol 2010; 10: 17.

11 Bohannon RW, Smith MB. Interrater reliability of a modified Ashworth scale of muscle spasticity. Phys Ther 1987; 67: 206-207.

12 Medical Research Council. Aids to the examination of the peripheral nervous system, Memorandum no. 45, Her Majesty's Stationery Office, London, 1981.

13 Ashburner J, Chen CC, Flandin G, Henson R, Kiebel S, Kilner J et al Statistical Parametric Mapping v.8. The FIL Methods Group: London, Uk. http://www.fil.ion.ucl. ac.uk/spm/ (August, 2010).

14 International Consortium for Brain Mapping. ICBM 452 T1 Atlas, 2010. http:// www.loni.ucla.edu/ICBM/Downloads/Downloads 452T1.shtml (August, 2010).

15 Rorden C. MRIcro v. 1.40. Center for Advanced Brain Imaging: Atlanta, GA, USA, 2005

16 Maldjian JA, Laurienti PJ, Kraft RA, Burdette JB. An automated method for neuroanatomic and cytoarchitectonic atlas-based interrogation of fMRI data sets. Neurolmage 2003; 19: 1233-1239.

17 Maldjian JA, Laurienti PJ, Burdette JH. Precentral gyrus discrepancy in electronic versions of the Talairach Atlas. Neurolmage 2004; 21: 450-455.

18 Wilke M, Schmithorst VJ. A combined bootstrap/histogram analysis approach for computing a lateralization index from neuroimaging data. Neuroimage 2006; 33: 522-530.

19 Kepler J, Kepler K, Tomberg T, Ilves P. Uncertainty in functional magnetic resonance imaging methods for cortex motor and language area examinations. In: Medical Physics in the Baltic States: 8th International Conference on Medical Physics; Kaunas, Lithuania; 14-16 October 2010. Kaunas Technol 2010; 2: 95-100.

20 Braschinsky M, Parts K, Maamägi H, Gross-Paju K, Haldre S. Functional assessment of lower extremities in hereditary spastic paraplegia. Arch Phys Med Rehabil 2009; 90 1887-1890.

21 Sartucci F, Tovani S, Murri L, Sagliocco L. Motor and somatosensory evoked potentials in Autosomal Dominant Hereditary Spastic Paraparesis (ADHSP) linked to chromosome 2p, SPG4. Brain Res Bull 2007; 74: 243-249.

22 Tallaksen CM, Dürr A, Brice A. Recent advances in hereditary spastic paraplegia. Cur Opin in Neurol 2001; 14: 457-463.

23 Konrad C. Brain plasticity and functional reorganization in progressive motor system degeneration. J Neurol Sci 2006; 244: 3-4. 